Cahiers $d u$ MONDE RUSSE

\section{Cahiers du monde russe}

Russie - Empire russe - Union soviétique et États indépendants

46/1-2 | 2005

La Russie vers 1550

\title{
La monarchie russe à la lumière de la crise politique des années 1530-1540
}

\section{Mohail M. KROM}

\section{OpenEdition}

\section{Journals}

Édition électronique

URL : https://journals.openedition.org/monderusse/8795

DOI : 10.4000/monderusse. 8795

ISSN : $1777-5388$

\section{Éditeur}

Éditions de l'EHESS

\section{Édition imprimée}

Date de publication : 1 janvier 2005

Pagination : 211-218

ISBN : 2-7132-2055-6

ISSN : $1252-6576$

Référence électronique

Mohail M. KROM, «La monarchie russe à la lumière de la crise politique des années 1530-1540», Cahiers du monde russe [En ligne], 46/1-2 | 2005, mis en ligne le 01 janvier 2007, consulté le 03 septembre 2022. URL : http://journals.openedition.org/monderusse/8795 ; DOI : https://doi.org/ 10.4000/monderusse.8795 
chercher : repérer : avancer

Cet article est disponible en ligne à l'adresse :

http://www.cairn.info/article.php?ID REVUE=CMR\&ID NUMPUBLIE=CMR 461\&ID ARTICLE=CMR 4610211

La monarchie russe à la lumière de la crise politique des années 1530-1540

par Mohail M. KROM

Editions de I'EHESS | Cahiers du monde russe

2005/1-2 - Vol 46

ISSN 1252-6576 | ISBN 2713220556 | pages 211 à 218

Pour citer cet article :

-M. KROM M., La monarchie russe à la lumière de la crise politique des années 1530-1540, Cahiers du monde russe 2005/ 1-2, Vol 46, p. 211-218.

Distribution électronique Cairn pour les Editions de l'EHESS.

(C) Editions de l'EHESS. Tous droits réservés pour tous pays.

La reproduction ou représentation de cet article, notamment par photocopie, n'est autorisée que dans les limites des conditions générales d'utilisation du site ou, le cas échéant, des conditions générales de la licence souscrite par votre établissement. Toute autre reproduction ou représentation, en tout ou partie, sous quelque forme et de quelque manière que ce soit, est interdite sauf accord préalable et écrit de l'éditeur, en dehors des cas prévus par la législation en vigueur en France. Il est précisé que son stockage dans une base de données est également interdit. 


\section{LA MONARCHIE RUSSE À LA LUMIÈRE DE LA CRISE POLITIQUE DES ANNÉES 1530-1540}

L'hypothèse principale sur laquelle repose ce travail est la suivante : durant la période dite du «gouvernement des bojare»(de 1533 à 1547 ou 1548, cette dernière date demandant à être précisée), le pays traversa une longue crise politique provoquée par l'incapacité de fait du jeune souverain Ivan $\mathrm{IV}^{1}$. De là découle le projet de cette recherche : étudier le fonctionnement de la «monarchie sans monarque » pour tenter de comprendre quelques particularités essentielles de la nature de l'État russe du Moyen Âge tardif.

\section{Fonctions du monarque et de ses conseillers dans le gouvernement de l'État}

Les périodes de crise sont pour les chercheurs comme des expériences proposées par l'histoire elle-même : ce qui demeure « hors champ » durant les périodes de stabilité politique passe au premier plan, devient visible durant les périodes de crise. Les historiens peinent à comprendre le mécanisme de prise de décision dans les situations «normales », lorsqu'un grand-prince ou un tsar adulte et majeur

1. Plusieurs articles de l'auteur sont consacrés à la justification de cette thèse. Voir М. М. Кром, « Политический кризис 30-40-х годов XVI века (Постановка проблемы) » [M. M. Krom, , «La crise politique des années 1530-1540 (position du problème)»], Отечественная история [Histoire nationale], 1998, n 5, p. 3-19 ; idem, "Мне сиротствующу, а царству вдовствующу": кризис власти и механизм принятия решений в период “боярского правления” (30-40-е годы XVI в.) », Российская монархия: вопросы истории и теории. Межвузовский сборник статей, посвященный 450-летию учреждения царства в России (1547-1997 г2.) [ "Je suis orphelin et l'Empire est veuf” : la crise du pouvoir et le mécanisme de prise de décision pendant le "gouvernement des bojare" (années 1530-1540)», in : La monarchie russe : questions d'histoire et de théorie. Recueil interuniversitaire d'articles consacrés au $450^{\mathrm{e}}$ anniversaire de l'institution impériale en Russie (1547-1997)], Voronež, 1998, p. 40-49. 
exerce le pouvoir. Que décidait le souverain lui-même, qu'est-ce qui émanait de ses conseillers ? Il est un peu plus facile de répondre à ces questions pour ce qui est du « gouvernement des bojare », c'est-à-dire de la période d'incapacité de fait d'Ivan IV.

La crise des années 1530-1540 mit en lumière de manière éclatante une fonction essentielle du monarque : celle de garant de la stabilité politique et d'arbitre suprême de l'élite de la cour. Ce fut précisément l'incapacité du jeune Ivan IV à assumer cette fonction qui engendra les explosions de violence et la multiplication sans précédent des querelles de préséance. Tant que sa mère, la grande-princesse Hélène Glinskaja, qui s'était approprié le titre de souveraine, était en vie, ces conflits étaient encore contenus tant bien que mal. Mais, après sa mort (avril 1538), l'aristocratie se sentit affranchie de tout contrôle. Pour la période comprise entre 1539 et la fin de l'année 1547 , le répertoire chronologique établi par Jurij Moiseevič Êskin enregistre 43 affaires de préséances, alors que 13 cas seulement sont connus pour tout le règne de Basile III (1505-1533) 2 .

Une autre prérogative imprescriptible du monarque était de représenter l'État en matière de politique extérieure. Il incarnait dans sa personne l'idée même de souveraineté. C'était lui, et nul autre, qui menait la guerre contre ses voisins et concluait la paix avec eux. En principe, cette fonction ne dépendait pas de l'âge ou des qualités physiques du détenteur du pouvoir suprême. Le bojarin Ivan Fedorovič Ovčina Obolenskij, le tout-puissant favori (et l'un des véritables responsables de la politique extérieure du pays au milieu des années 1530) a su exprimer cette idée de façon remarquable. Répondant à l'hetman lituanien Jerzy Radziwiłł, qui avait souligné dans une de ses lettres la jeunesse d'Ivan IV, il affirma : « ...notre souverain est encore dans l'enfance, mais majeur par la grâce de Dieu pour ce qui est de sa souveraineté $»^{3}$. (On peut voir dans cette formule une variante russe de la célèbre théorie médiévale des « deux corps du roi ») ${ }^{4}$. En décembre 1533, Ivan IV, alors âgé de trois ans, prit part pour la première fois à une cérémonie aulique (la réception de courriers envoyés de Crimée). Par la suite, l'audience accordée par le jeune souverain demeura un rituel obligatoire pour toutes les ambassades envoyées à Moscou$^{5}$.

Si l'on veut savoir qui expédiait les affaires courantes et prenait les décisions, il faut chercher la réponse dans les annotations de chancellerie au verso des chartes de privilèges. Bien qu'elles aient été, selon la tradition, délivrées au nom du grand-

2. Voir Ю. М. Эскин, Местничество в России XVI-XVII вв. Хронологический реестр [Ju. M. Êskin, Les querelles de préséance en Russie, $X V I^{e}-X V I I^{e}$ siècles. Répertoire chronologique], Moscou, 1994, p. 43-47, n 36-77 (juin 1539-décembre 1547); ibid., p. 41-42, n 20-32 (1506/07-1530).

3. « Государь наш ныне во младых летех, а милостью Божиею государствы своими в совершенных летех », Sb. RIO, t. 59, Saint-Pétersbourg, 1887, p. 37.

4. E. Kantorowicz, The King's Two Bodies. A Study in Medieval Political Theology, Princeton, 1957.

5. RGADA, fonds 123, dossier 8, f. 3, $33 \mathrm{v}^{\circ}, 160-160 \mathrm{v}^{\circ}$., etc. ; Sb. RIO, t. 59, 1887, p. 43-44 ;

Б. Н. Флоря, Иван Грозный [B. N. Florja, Ivan le Terrible], Moscou, 1999, p. 9. 
prince, ces chartes portent souvent au verso le nom de celui qui les a fait établir. Ainsi, au dos du privilège octroyé en janvier 1535 par Ivan IV au monastère de la Trinité Mahriščskij pour un hameau du district de Perejaslavl'-Zalesskij, on lit: «Sur l'ordre du bojarin prince Ivan Vasil'evič Šujskij » 6 . Deux autres chartes délivrées sur l'ordre du prince Ivan Vasil'evič Šujskij (en 1538)7 nous sont parvenues. Mais, la plupart du temps, on trouve au verso des chartes des noms de maîtres de l'Hôtel (двореикие) et de trésoriers. Sur les 54 chartes présentant des inscriptions au verso répertoriées pour la période 1535-1548, 48 ont été délivrées sur l'ordre de maîtres de l'Hôtel de Moscou (Большой дворец) ou de province et de trésoriers ${ }^{8}$. Il ne s'agissait d'ailleurs pas d'une pratique nouvelle : certaines chartes émises sous Basile III portent des mentions semblables ${ }^{9}$. Cela incite à penser que l'administration au jour le jour de la maison du grand-prince pouvait parfaitement se passer de l'intervention personnelle de celui-ci. Ce fut le cas dans les années 1530-1540. Lorsque, fait extrêmement rare, le souverain prenait lui-même la décision, les secrétaires ne manquaient pas de le mentionner. Ainsi, le privilège d'Ivan IV du 6 février 1545 accordé au monastère Saint-Antoine de la Sija porte l'annotation : «C'est le grand-prince lui-même qui a donné l'ordre de rédiger cette nouvelle charte. [signé :] Istoma Nougorodov $»^{10}$.

À en juger par les documents qui ont été conservés, l'intervention personnelle du jeune souverain était bien plus fréquente dans les affaires de justice. Ainsi, entre février 1535 et juin 1536, il entendit plusieurs minutes de procès et rendit son verdict en conséquence ${ }^{11}$. Si l'on garde à l'esprit que le grand-prince n'était alors âgé que de quatre à cinq ans, il est évident que son rôle dans la procédure judiciaire était purement nominal. Les cas où les affaires judiciaires étaient réglées à Moscou par des bojare qui n'appartenaient pas à l'administration du palais sont réellement exceptionnels (on peut une fois de plus citer ici le prince Ivan Vasil’evič Šujskij) ${ }^{12}$.

6. RNB, Département des manuscrits, fonds 532, inventaire $1, \mathrm{n}^{\circ} 113$.

7. Описание актов собрания графа А. С. Уварова. Акты исторические, описанные И. М. Катаевым и А. К. Кабановым [Inventaire des actes de la collection du comte A. S. Uvarov. Documents historiques inventoriés par I. M. Kataev et A. K. Kabanov], Moscou, $1905, n^{\circ} 25$, p. 32 ; RGB, Département des manuscrits, fonds 303, registre 536, f. 432-432 vº.

8. Le trésorier Ivan Ivanovič Tret jakov (seul ou avec un autre trésorier, Fedor Ivanovič Sukin) fut à l'origine de 27 chartes; le maître de l'Hôtel de Moscou, le prince Ivan Ivanovič Kubenskij, de 7, etc. Les indications au verso des chartes sur l'identité de ceux qui les firent émettre furent publiées pour la première fois par S. M. Kaštanov dans son répertoire chronologique. Voir C. М. Каштанов, “Хронологический перечень иммунитетных грамот XVI века [Ч. 1], [S. M. Kaštanov, « Répertoire chronologique des privilèges du XVI siècle [1 ${ }^{\text {re }}$ partie] »], AE 1957, Moscou, 1958, p. 302-376. En ce qui concerne les années 1530-1540, voir les n 343, $345,379,402,415,419,426,427,433,441$, etc.

9. Ibid., $\mathrm{n}^{\circ} 44$ (1507), 63 (1508), 99 (1511), etc.

10. SGKÊE, t. I, Petrograd, $1922, \mathrm{n}^{\circ} 109$, col. 112.

11. RGB, Département des manuscrits, fonds 303, registre 518, f. 341-341 v ; AGR, t. I, Kiev, 1860, p. 46-47, 49-52, 55-57.

12. Le 4 mai 1534, le prince Ivan Vasil’evič Šujskij rendit un jugement en faveur du monastère Mahriščskij dans le différend qui l'opposait aux Baskakovy ( $A J u B$, t. I, Saint-Pétersbourg, 1857, n 52, col. 172-192). 
On peut considérer que, dans les années 1530-1540, l'habitude était que les maîtres d'Hôtel de Moscou et de province et les trésoriers officient comme juges de dernière instance ${ }^{13}$.

\section{Haute « politique » et routine administrative}

Tout ce que recouvre pour nous aujourd'hui le terme «politique » (ce mot était inconnu dans la Russie du XVI ${ }^{\text {e }}$ siècle) était rangé par les gens de cette époque dans deux sphères différentes et de valeurs très inégales. D'une part, la haute sphère, qui englobait tout ce qui concernait la personne du souverain, ses faits et gestes (par exemple, la guerre était bien évidemment «l'affaire du souverain »), ainsi que les événements de la vie de cour. D'autre part, la routine administrative, le fonctionnement au jour le jour des bureaux. Le contraste entre ces deux sphères est facile à repérer si nous comparons les documents de la pratique administrative mentionnés ci-dessus avec les récits des chroniques sur l'époque du «gouvernement des bojare ». Parlant des guerres et des relations diplomatiques avec les États voisins, des intrigues de la cour et des révolutions de palais au Kremlin, les chroniqueurs ne disent pratiquement rien des affaires ni des préoccupations d'administration intérieure. Ils ne nous renseignent même pas sur des mesures aussi essentielles que la réforme de l'administration locale de la justice et de la police [губная реформа] et l'attribution de bénéfices, ou domaines en précaire [поместья], à la fin des années $1530^{14}$.

La vie de la cour, qui était au centre de l'attention des chroniqueurs, était une sorte de «théâtre politique », où tous les personnages se trouvaient en permanence en représentation : le grand-prince, sa mère la régente, ses oncles (qui mouraient les uns après les autres en détention), les bojare favoris, les métropolites. Cependant, les rouages de l'administration quotidienne du pays étaient actionnés par d'autres personnages, bien plus rarement mentionnés dans les chroniques : les maîtres de l'Hôtel, les trésoriers, les secrétaires. Naturellement, il existait des recoupements entre les sphères de la cour et de l'administration : d'une part, certains favoris, comme les princes Ivan Fedorovič Ovčina Obolenskij et Ivan Vasil'evič Šujskij, endossèrent des fonctions judiciaires et administratives ; d'autre part, des administrateurs expérimentés comme le prince Ivan Ivanovič Kubenskij, maître de l'Hôtel, et le secrétaire Fedor Mišurin furent impliqués dans des intrigues de cour et finirent sur le billot. Mais elles ne coïncidaient que partiellement. Il est indispensable de distinguer ces deux sphères pour comprendre les événements des années 15301540 : la crise toucha surtout la sphère du pouvoir suprême et n'eut presque aucun

13. AGR, p. 81-82, 84-85, 91-92, 103-110 ; RGADA, fonds 1193, registre 1, f. 104 ; RGB, Département des manuscrits, fonds 303, registre 539, f. 273a-274; ASZ, t. I, Moscou, 1997, $\mathrm{n}^{\circ} 122,228,229,314$, p. 96, 202, 206, 311, etc.

14. On ne trouve d'informations sur la réforme de l'administration locale que dans les chroniques de Pskov. Voir Псковские летописи [Les chroniques de Pskov], vol. 1, Moscou-Leningrad, 1941, p. 110 ; vol. 2, Moscou, 1955, p. 229-230. 
effet sur l'appareil étatique en tant que tel. Or, les intérêts de la noblesse étaient presque entièrement concentrés dans cette sphère « supérieure », celle de la cour. C'est justement pour cette raison que les conflits au sein de l'aristocratie, qui se traduisaient par des querelles de préséance, n'eurent pas de conséquences destructrices sur l'appareil administratif et n'engendrèrent pas l'anarchie dans le pays.

Ce qui m'amène à penser que l'idée, solidement ancrée pourtant dans l'historiographie, selon laquelle l'émergence d'un nouveau favori signifiait un changement de gouvernement, est totalement erronée. Entre 1538 et 1547, cinq « gouvernements » en tout se seraient succédé, correspondant chacun à un groupe de bojare : les Šujskie, les Bel'skie, les Voroncovy, les Glinskie, etc. ${ }^{15}$ Dans cette conception, la vie politique du XVI siècle est pensée par analogie avec la vie des États du XIXe et du $\mathrm{XX}^{\mathrm{e}}$ siècle (avec leurs changements de cabinets, les « chassés-croisés » ministériels, etc.). Mais les groupes de bojare du XVI ${ }^{\mathrm{e}}$ siècle n'avaient rien de commun avec les partis politiques modernes. Ils ne cherchaient nullement à «s'emparer de l'appareil d'État » (comme l'ont affirmé certains historiens $)^{16}$, à modifier la politique gouvernementale, à mettre en œuvre des réformes. L'objectif des clans de bojare qui s'affrontaient était de s'assurer une situation dominante au sein de la cour, de distribuer les grades du Conseil à leurs parents et partisans et probablement d'améliorer leur situation matérielle aux dépens du trésor.

Les bojare, on l'a montré plus haut, ne prenaient part qu'épisodiquement aux activités administratives et judiciaires. Aucune des tentatives faites par les historiens pour attribuer à tel ou tel favori un programme politique déterminé n'est convaincante. Par «gouvernement» (si tant est que ce terme puisse être employé ici) il faut plutôt entendre d'autres personnages, ceux qui quotidiennement - on peut déjà dire professionnellement - assuraient la bonne marche de l'administration : les maîtres de l'Hôtel, les trésoriers, les secrétaires, qui constituaient un embryon de bureaucratie. La lutte contre le brigandage offre, à cet égard, un exemple significatif. À la charnière des années 1530 et 1540 , ce sont des nobles issus de divers clans de princes et de bojare (dans l'ordre chronologique : le prince Ivan Dmitrievič Penkov, le prince Ivan Vasil'evič Šujskij et Ivan Grigor'evič

15. I. I. Smirnov parle de la « politique des Bel’skie », du « gouvernement des Bel`skie », etc., mais ne va pas jusqu'à identifier tel ou tel groupe de bojare avec le gouvernement du pays. La tendance à l'identification est en revanche clairement exprimée dans le livre d'A. A. Zimin : « la politique du gouvernement des Bel'skie » (au sujet des années 1540-1541) et « le nouveau gouvernement » (à propos de l'émergence des Voroncovy et des Kubenskie en 1544-1545). Il faut attendre l'ouvrage de S. M. Kaštanov pour trouver un tableau achevé de la valse des «gouvernements» des bojare, chacun essayant de mener sa propre politique. Voir И. И. Смирнов, Очерки политической истории Русского государства 30-50-х годов XVI века [I. I. Smirnov, Essais sur 1'histoire politique de la Russie, 1530-1560], Moscou, 1958, p. 75119, en particulier p. 86, 93,113 ; А. А. Зимин, Реформы Ивана Грозного [A. A. Zimin, Les réformes d'Ivan le Terrible], Moscou, 1960, p. 248-278, en particulier p. 258, 268 ; C. М. Каштанов, Социально-политическая история России конца XV-первой половинь XVI в. [S. M. Kaštanov, Histoire socio-politique de la Russie, fin du XVe-première moitié du XVI siècle], Moscou, 1967, p. 327-374.

16. A. A. Zimin, Les réformes d'Ivan le Terrible (op. cit., note 15), p. 224. Cette thèse reçut le soutien de V. B. Kobrin dans son compte-rendu du livre d'A. A. Zimin (voir История СССР [Histoire de 1'URSS], 1962, n 5, p. 186). 
Morozov) qui étaient chargés de la diriger. Mais ces changements de personnes n'eurent aucune incidence sur le caractère de la réforme judiciaire, probablement parce que toutes les chartes relatives à l'administration locale de la justice et de la police [губные грамоты] émanaient d'une même administration. Comme l'a montré Nikolaj Evgen'evič Nosov, elles étaient signées par les secrétaires du palais (дворизовые дьяки $)^{17}$.

\section{La monarchie russe dans une perspective historique comparative}

Si l'on compare la crise politique traversée par la Russie dans les années 1530-1540 avec la situation d'autres pays européens au début des Temps modernes, on remarque que partout la minorité du souverain mettait en évidence la faiblesse institutionnelle de la monarchie. L'ensemble des problèmes auxquels était confronté le pouvoir suprême était similaire : une élite politique difficile à diriger, les prétentions des princes de sang, les dissensions au sein de la famille royale, etc. ${ }^{18} \mathrm{La}$ monarchie d'Ivan IV présente cependant certaines particularités de caractère nettement archaïque et oblige à chercher des parallèles dans un passé européen plus reculé.

Il s'agit d'abord du fait que l'institution de la régence n'avait pas de bases juridiques dans la Russie du XVI siècle. Au Moyen Âge, bien que le roi de France fût pleinement en droit de choisir comme tuteur de son fils mineur la personne en qui il avait le plus confiance (la mère du prince héritier, un de ses oncles, une sœur, etc.) et qu'il n'y eût en cette matière aucun règlement précis ${ }^{19}$, l'institution même de la régence existait et n'était remise en cause par personne. En Russie, bien que la tradition de la tutelle familiale fût bien connue dans la lignée grand-princière, la notion de régence n'existait pas en tant que telle. L'intégralité du pouvoir appartenait officiellement au souverain, indépendamment de son âge (gardons à l'esprit les propos cités plus haut d'Ivan Fedorovič Ovčina Obolenskij), et ne pouvait être transmise à personne. Même la régence d'Hélène Glinskaja, qui avait le droit incontestable de tutelle sur son fils, revêtait pour ainsi dire un caractère semi-officiel. Elle ne pouvait ouvertement intervenir au nom d'Ivan IV ni dans le domaine judiciaire, ni dans l'administration intérieure, ni dans la politique étrangère ${ }^{20}$. Les prétendants ultérieurs au rôle de tuteur du jeune souverain étaient encore moins en

17. Н. Е. Носов, Очерки по истории местного управления Русского государства первой половины XVI века [N. E. Nosov, Essais sur l'histoire de l'administration locale en Russie dans la première moitié du XVI siècle], Moscou-Leningrad, 1957, p. 315-326.

18. N. Henshall, The Myth of Absolutism. Change and Continuity in Early Modern European Monarchy, Londres - New York, 1992. Cité ici d'après l'édition russe : Н. Хеншелл, Миф абсолютизма. Перемены и преемственность в развитии западноевропейской монархии раннего Нового времени, Saint-Pétersbourg, 2003, p. 25-26, 40, 172.

19. Dictionnaire de la France médiévale, sous la direction de Michel Balard, Paris, 2003, p. 226 (article « régent »).

20. Voir M. M. Krom, « La crise politique... » (op . cit., note 1), p. 7. 
droit de se considérer comme les dirigeants légitimes du pays, et ce fut l'un des principaux facteurs d'instabilité à la fin des années 1530 et dans les années 1540 .

La situation décrite rappelle l'Europe du haut Moyen Âge, où la capacité juridique d'un roi mineur n'était en rien limitée et où il n'existait pas de tuteurs ni de régents officiellement désignés qui auraient gouverné à la place du monarque ${ }^{21}$.

De même, le début de bureaucratisation du gouvernement de la Moscovie dans le deuxième quart du XVI ${ }^{\mathrm{e}}$ siècle rappelle davantage la France des XII ${ }^{\mathrm{e}}$-XIII ${ }^{\mathrm{e}}$ siècles que les États européens du début des Temps modernes. Nous avons gardé la trace (originaux, copies ou simples mentions) de plus de 400 privilèges et mandements émis au nom d'Ivan IV et datant du « gouvernement des bojare », conservés pour la plupart dans des archives monastiques ${ }^{22}$. Il y faut ajouter plusieurs dizaines de documents divers (minutes de procès, chartes statutaires, chartes relatives à l'administration locale, etc.). De très nombreux documents ont évidemment disparu dans les incendies et autres catastrophes, mais les archives des autres pays n'y ont pas échappé non plus : de ce point de vue, la situation russe est loin d'être unique. Même en tenant compte de ces pertes, la « productivité » de la chancellerie grandprincière moscovite des années 1530-1540 est de beaucoup inférieure à celle de la chancellerie royale de Philippe IV le Bel (1285-1314), dont pas moins de 50000 actes sont parvenus jusqu'à nous. Ainsi que le souligne Robert Fawtier à qui je dois ce chiffre, la quantité de documents conservés «n'est rien auprès de ce qui a été détruit ${ }^{23}$. Il est évident que les deux États en sont à des étapes différentes de leur développement. En effet, le premier document de la chancellerie moscovite que nous connaissions est le testament d'Ivan Kalita ( « l'Escarcelle ») daté de 1340.

La quantité des actes est habituellement mise en corrélation avec les effectifs du personnel administratif. À cet égard, le contraste entre la Russie et la France du XVI siècle est saisissant: Aleksandr Aleksandrovič Zimin a compté 121 secrétaires au service du grand-prince Basile III pour l'ensemble de son règne (1505-1533) ${ }^{24}$; lors de son accession au trône, François Ier (1515-1547) disposait d'une véritable armée d'officiers, estimée à 4000 personnes (en 1573, ils seront déjà 20 000)25.

21. Th. Offergeld, Reges pueri. Das Königtum Minderjähriger im frühen Mittelalter, Hannovre, 2001, p. 35.

22. Dans S. M. Kaštanov, « Répertoire chronologique... » (op . cit., note 8), ainsi que dans les compléments édités en 1968 (С. М. Каштанов, В.Д. Назаров, Б. Н. Флоря, «Хронологический перечень иммунитетных грамот XVI в. [Ч. 3]» [S. M. Kaštanov, V.D. Nazarov, B. N. Florja, «Répertoire chronologique des privilèges du XVI siècle [3e partie] »], AE 1966, Moscou, 1968, p. 197-253), pour les années 1534-1548 sont recensés 409 privilèges. Les recherches menées au cours des trente dernières années permettent certainement d'ajouter à ce nombre plusieurs dizaines de documents.

23. R. Fawtier, Les Capétiens et la France, Paris, 1942, p. 14 (cité par l'auteur d'après la traduction russe, Saint-Pétersbourg, 2001).

24. Voir A. А. Зимин, “Дьяческий аппарат в России второй половины XV- первой трети XVI в.”, Исторические записки [A. A. Zimin, « Le corps des secrétaires dans la Russie de la seconde moitié du XVe siècle et le premier tiers du XVI ${ }^{\mathrm{e}}$ », Notes historiques], vol. 87, Moscou, 1971, p. 282.

25. F. Cosandey, R. Descimon, L'absolutisme en France. Histoire et historiographie, Paris, 2002, p. 110. 
Cet archaïsme de la monarchie russe, sensible si on la compare aux royaumes d'Europe occidentale de la même époque, ne doit cependant pas être interprété comme un signe d'immobilisme et de stagnation. Les années 1530-1540 sont précisément dans l'histoire de la Russie une période de changements profonds du système d'administration centrale et locale, où se poursuit la bureaucratisation de l'appareil administratif. Le Justicier de 1550 reflète ces changements ${ }^{26}$.

(traduit du russe par Élisabeth Teiro et André Berelowitch)

Université européenne de Saint-Pétersbourg

Faculté d'histoire

krom@eu.spb.ru

26. Voir M. М. Кром, “Судебник 1550 г. и судебно-административная практика 30-40-х годов XVI в.” [ « Le Justicier de 1550 confronté à la pratique administrative et judiciaire des années 1530 et $1540 »]$, Восточная Европа в древности и средневековье. Проблемы источниковедения (XVII Чтения памяти В. T. Пашуто; IV Чтения памяти A. А. Зимина) [L'Europe orientale dans l'Antiquité et au Moyen Âge. Problèmes d'étude des sources $\left(X V I I I^{e}\right.$ conférence à la mémoire de V. T. Pašuto; IVe conférence à la mémoire de A. A. Zimin], vol. II, Moscou, 2005, p. 217-220. 